\title{
Ridge Detection and Analysis of Susceptibility-Weighted Magnetic Resonance Imaging in neonatal Hypoxic- Ischaemic Encephalopathy
}

\author{
Zhen Tang ${ }^{1}$, Sasan Mahmoodi ${ }^{1}$, Srinandan Dasmahapatra ${ }^{1}$, Angela Darekar ${ }^{2}$, Brigitte \\ Vollmer $^{3}$ \\ ${ }^{1}$ Electronics and Computer Science, University of Southampton, University Road, Southamp- \\ ton, Southampton, United Kingdom, SO17 1BJ \\ ${ }^{2}$ Department of Medical Physics, University Hospital Southampton NHS Foundation Trust, \\ Tremona Road, Southampton, United Kingdom, SO16 6YD \\ ${ }^{3}$ Clinical Neurosciences, Clinical and Experimental Sciences, Faculty of Medicine, Univer- \\ sity of Southampton, Southampton, United Kingdom, SO17 1BJ \\ zt1n18@soton.ac.uk, sm3@ecs.soton.ac.uk, sd@ecs.soton.ac.uk, \\ Angela.Darekareuhs.nhs.uk, B.Vollmer@soton.ac.uk
}

\begin{abstract}
The purpose of this study is to develop a new automated system to classify susceptibility weighted images (SWI) obtained to evaluate neonatal hypoxic-ischaemic injury, by detecting and analyzing ridges within these images. SW images can depict abnormal cerebral venous contrast as a consequence of abnormal blood flow, perfusion and thus oxygenation in babies with HIE. In this research, a dataset of SWI-MRI images, acquired from 42 infants with HIE during the neonatal period, features are obtained based on ridge analysis of SW images including the width of blood vessels, the change in intensity of the veins' pixels in comparison with neighboring pixels, the length of blood vessels and Hessian eigenvalues for ridges are extracted. Normalized histogram parameters in the single or combined features are used to classify SWIs by $k N N$ and random forest classifiers. The mean and standard deviation of the classification accuracies are derived by randomly selecting 11 datasets ten times from those with normal neurological outcome $(n=31)$ at age 24 months and those with abnormal neurological outcome $(n=11)$, to avoids classification biases due to any imbalanced data. The feature vectors containing width, intensity, length and eigenvalue show a promising classification accuracy of $78.67 \% \pm 2.58 \%$. The features derived from the ridges of the blood vessels have a good discriminative power for prediction of neurological outcome in infants with neonatal HIE. We also employ Support Vector Regression (SVR) to predict the scores of motor and cognitive outcomes assessed 24 months after the birth. Our mean relative errors for cognitive and motor outcome scores are $0.113 \pm 0.13$ and $0.109 \pm 0.067$ respectively.
\end{abstract}

Keywords: Hypoxic-Ischaemic Encephalopathy, SWI Ridges, neurological outcome, vessel intensity 


\section{Introduction}

Hypoxic-ischaemic encephalopathy (HIE) as a consequence of perinatal asphyxia, is an important cause of perinatal death and neurodevelopmental abnormality in neonates worldwide [1]. There are around $0.2 \%$ infants affected by HIE in developed countries. And 20 and 50 percent of asphyxiated newborns show hypoxic-ischemic encephalopathy, which die in the neonatal period. As many as 25 percent of survivors show permanent neuropsychological disorders. Early diagnosis and intervention can improve the prognosis of HIE and reduce the occurrence of sequelae [3]. Magnetic resonance image (MRI) is an important tool for the assessment of hypoxic-ischaemic injury and for aiding early prediction of neurodevelopmental outcomes [4].

Susceptibility weighted imaging (SWI) can significantly increase the sensitivity and specificity of the diagnosis of neurological diseases in children [5]. The technique employs a 3D T2* weighted sequence with high spatial resolution and flow compensation [6]. This sequence is very sensitive to the detection of blood oxygenation level changes within the venous vasculature, due to the increased magnetic susceptibility of deoxygenated blood. It is thus useful in detecting these changes in relation to hypoxia or ischemia in the brain [7]. Therefore, it is a promising sequence, which, when used in conjunction with conventional T1-weighted (w) and T2-w images, as well as Diffusion Weighted Imaging (DWI) [8-9], could improve the predictive value of neonatal MRI in HIE. In [10], a machine learning tool is developed to predict outcomes for neonatal HIE patients by building their Apparent Diffusion Coefficient (ADC) map measurements. In [11], the set of Hessian eigenvalues of the ridges in the SWIs is used to correctly diagnose infants for HIE. They were divided into two categories based on the radiological report of the scan [12] -10 healthy infants and 48 infants with HIE, and the best classification accuracy was reported in [11] to be $91.83 \%$. In [13], an extended three-dimensional local binary patterns (LBP) is developed to distinguish the oxygenated images of three-dimensional SW images of infants with HIE, thought to be related to the oxygenation levels of the blood - a possible marker of hypoxic ischemic injury. A total of 17 individuals with their SWIs is considered in [13] where seven of them are affected by HIE. The best classification accuracy reported in [13] is $89.9 \%$. However, there are two issues related to the classification accuracies presented in [11] [13]. 1) The classification accuracies are calculated in [11] and [13] by using Kitamura scores evaluated by radiologists. 2) The data in these studies is unbalanced. Therefore, the accuracies reported in these works are somewhat biased.

In this paper, we use a balanced dataset of neonatal SWI MR images and employ the neurological outcomes of patients at the age of 24 months as the outcome measure. We therefore present an automated HIE prognosis system, by using four feature vectors: histograms of the width, intensity, length and the largest eigenvalue of Hessian of vessels detected as ridges to predict neurological outcomes. A ridge detection method is used for vessel detection and the vessels are segmented in the ridge images to remove undesirable segmented noise and nonvascular segments. We manually segment 5 slices as ground truth to inspect the error in vessel segmentation. Pixels on the Canny images of original SWI and detected ridges are employed to obtain the width, intensity, length and the largest Hessian eigenvalue. The histograms of these four features are calculated 
for each dataset as feature vectors and SWIs are classified by these parameters from these histograms. Cognitive and motor developments of patients at age 24 months could be assessed by Bayley Scales, which result in a scaled score that can be used as a continuous variable to make regression model to predict the scores measured for the patients. We use Support Vector Regression (SVR) to predict the cognitive and motor scores of infants from four feature vectors. This paper is structured as follows: The methods we have used are described in section 3. Our numerical analysis and results are presented in section 4 . The analysis and results of our regression model are detailed in section 5 and finally we draw conclusions in section 6 .

\section{Ethics:}

We use 42 data sets of infants with neonatal HIE. All infants born at GA $>36+6$ weeks who underwent hypothermia treatment are scanned on a $1.5 \mathrm{~T}$ Siemens Symphony MRI scanner (median 7.8 [range 1-34] postnatal days), including Axial proton density (PD), T1-weighted, T2-weighted, turbo inversion recovery (IR), DWI and SWI. Pulse sequence parameters for SWI is left handed system, flow compensated, spoiled gradient echo (FLASH), TR $/ \mathrm{TE} / \alpha=50 \mathrm{~ms} / 40 \mathrm{~ms} / 12^{\circ}$ - long TE, voxel size $=0.9 \times 0.9 \times 2$ $\mathrm{mm}^{3}$, bandwidth $=70 \mathrm{~Hz} /$ pixel.

We have been granted permission to use the clinical scans for this dataset (HRA and Health and Care Research Wales (HCRW); IRAS ID 279072; REC reference 20/HRA/0260 as well as NRES Committee London - City \& East; IRAS ID 143392; REC reference 13/LO/1948; Protocol number 1). All infants have neurological assessment at age 24 months (outcome: normal neurology or abnormal neurology i.e. Cerebral Palsy, 11 infants (26.2\%) have Cerebral Palsy and 31 (73.8\%) have normal neurological outcome). Some of infants are assessed cognitive and motor developments by Bayley Scales as scaled scores at age 24 months, which result in a scaled score. (29 infants have cognitive scores, 28 have motor scores and the remaining infants have neither cognitive scores nor motor scores).

\section{Methods}

\subsection{Active Contour Model}

Susceptibility-weighted images are very sensitive to the detection of vascular extraneous blood products and hypoxic ischemia [11]. We used an active contour model to segment the brain and to remove the background from the SW images to enable us to focus more on the blood vessels in brain [14] as shown in Figure 2(b).

\subsection{Ridge Detection}

Since the SW images we used are two-dimensional slices, we applied zero-crossing to find ridges of vessels in SW images. First, scale-space representation can separate out 
information effectively. Image $I(x, y ; \sigma)$ can be obtained by convolving the image $I(x, y)$ with a Gaussian kernel $G(x, y ; \sigma)$ of variance $\sigma$ to reduce the noise:

$$
I(x, y ; \sigma)=I(x, y) * G(x, y ; \sigma)
$$

Let us denote $I(x, y)$ to be the SW image after segmenting the brain such as the one shown in Figure 2(b) by using active contours. The first derivative of the image $I(x, y)$ has a zero-crossing point that corresponds to the ridge. We can set a threshold value to remove the influence of noise. The center lines in brain which is non-vascular are removed by Hough Transform.

\subsection{Ridge Segmentation}

Vessel segmentation is a significant step after ridge detection. The ridge map image shown in Figure 2(d) is a binary image in which 1's (white pixels) represent ridges. Ridge maps are used in the connected component labelling algorithms [15-16] for ridge segmentation to label the white pixels (objects) in the binary image so that each individual connected region is assigned a label. We choose a $3 \times 3$ window for to check 8 connectivity, in which there are eight connected pixels around each pixel, so that these pixels have to share edge or vertex.

Before ridge segmentation, we need to detect the bifurcation and end points. Bifurcation points are the points in which one vessel splits into two vessels, and end points are the points in which a ridge (vessel) ends. Bifurcation points can be found by convolving the template shown in Figure 1(a) with a ridge map such as the one shown in Figure 2(d) and looking for maxima. Similarly end points can be detected by and looking for extrema in an image obtained by convolving a ridge map with all rotations of the template shown in Figure 1(b). Bifurcation points should be deleted in order to separate crossing vessels and end points are retained in the lists of pixels representing segmented ridges. Each blood vessel is therefore labeled differently and given a different colour as shown in Figure 2(e).

\begin{tabular}{|l|l|l|}
\hline 1 & 1 & 1 \\
\hline 1 & 10 & 1 \\
\hline 1 & 1 & 1 \\
\hline
\end{tabular}

(a)

\begin{tabular}{|l|l|l|}
\hline 0 & 0 & 0 \\
\hline 0 & 1 & 0 \\
\hline 0 & 0 & 1 \\
\hline
\end{tabular}

(b)

Fig. 1. (a) Bifurcation points template, where convolution with the ridge point is equal or greater than to 13. (b) End point template 


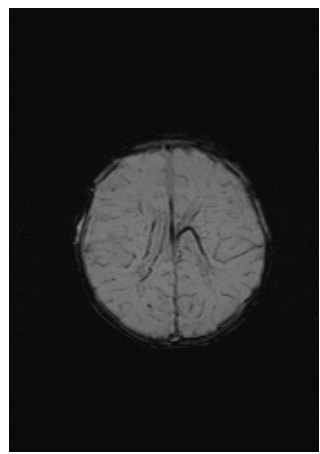

(a)

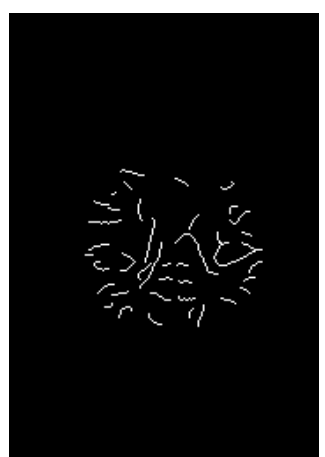

(d)

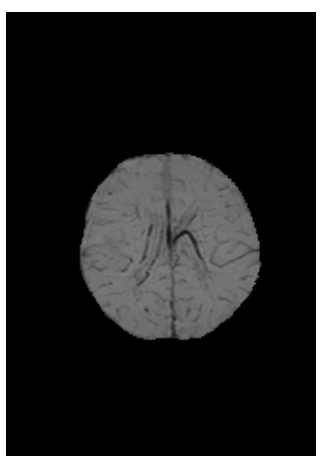

(b)

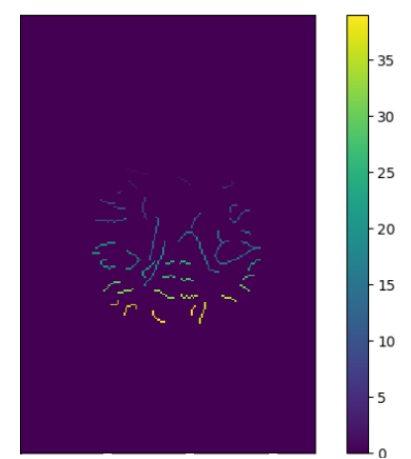

(e)

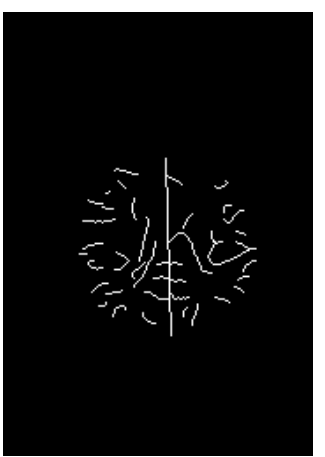

(c)

Fig. 2. (a) The original SW Image (b) The result after using active contour model (c) Ridge detection with gray value threshold $=40$ and threshold of difference between zero-crossing point and closest point is 270 . (d) The centerline of brain removed with pixel $=2$. (e) labelled blood vessel

\subsection{Feature Extraction}

Vessel width measurement. The locations of ridges can be obtained after ridge segmentation followed by Canny edge detection to find the edges of blood vessels. We use three consecutive points on the ridge (A, B and C) shown in Figure 3(a) and the line formed by connecting points $\mathrm{B}$ and $\mathrm{C}$ is known as ' $\mathrm{BC}$ ' here. On the edges of blood vessels, there are some intersections along a line normal to $\mathrm{BC}$ at point $\mathrm{A}$, shown in Figure 3(a). The minimum Euclidean distance of these intersection points is the vessel width. Finally, the histogram of all vessel widths is computed as a feature vector.

Vessel intensity measurement. In the SW images of infants with HIE, there will be various intensity values representing the gray scale values of blood vessels, which depict varying levels of blood oxygenation - thought to be a 'marker' of hemodynamic/vascular changes in brain due to HIE. Therefore, the difference between the pixel value of the ridge points and the edge points can be used as a feature for HIE outcome 
prognosis. In this paper, such a grayscale difference is referred to 'intensity'. We can obtain the coordinates of ridge points (A) and the corresponding two edge points, $\left(x_{1}, y_{1}\right)$ and $\left(x_{2}, y_{2}\right)$. By calculating respectively, the intensity difference of pixels on vessels and its boundary in the original grayscale image, the maximum difference is used as the vessel intensity. The histogram of all vessel intensities is computed as a feature vector. Figure 3(b-c) shows the vessel of an infant with HIE and the value of vessel pixels.

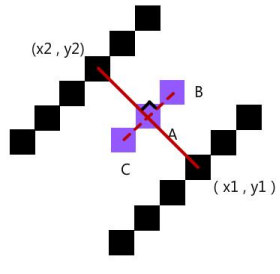

(a)

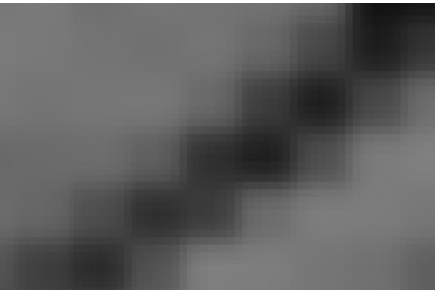

(b)

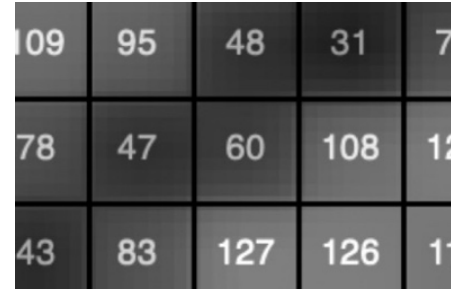

(c)

Fig. 3. (a) The pixels pairs according to vertical line. (b) Gray-scale SWI. (c) The value in gray-scale SW image.

Ridge Length Measurement. We also measure the length of blood vessels as a feature for HIE outcome prognosis. Having segmented the vessels, we count the number of pixels in each segmented vessel as a length measurement. A histogram of all vessel lengths constitutes a feature vector referred to length in this paper.

Ridge Eigenvalues. Ridge point is the point where the intensity image has a local minimum in the direction where the gradient changes the most [11]. The second derivative information can be derived from the Hessian matrix for the local intensities in the neighborhood of a pixel on the ridge. Therefore, the maximum of the absolute eigenvalue $(|\lambda|)$ of Hessian matrix of the points on the ridge are calculated as a feature. A histogram of such eigenvalues for every patient is thus considered as a feature vector.

\section{$4 \quad$ Results}

\subsection{Error of Vessel Segmentation}

We select 6 slices of SW images with the size $290 \times 256$ pixels equally per patient. Also, five slices of SW images from 5 infants are manually segmented as our ground truth data. We manually segment a total of 2455 pixels totally as vessels, and also apply our algorithm to these SW images to measure vessel segmentation errors. The difference in the number of pixels between manually segmented ridges and automatically segmented ridges in each slice is the number of error pixels, $n$. The error is normalized to be: 


$$
p=\frac{n}{m}
$$

where $m$ is the total number of the ground truth pixels of a vessel. According to the comparison in figure, the error pixels in these $5 \mathrm{SW}$ images are 187 pixels totally. We calculate the error accuracy in each of the five images and obtain a mean and standard deviation of the error to be $p=7.75 \pm 1.97 \%$ for our vessel segmentation method.

\subsection{Classification of results}

Having computed the histograms for width, intensity, length and Hessian eigenvalue of vessels in SWIs in our database, we classify the infants into two groups: infants with 1) abnormal neurological outcome (Cerebral Palsy) and 2) normal neurological outcome. In our dataset, the number of SWIs is imbalanced. There are 11 and 31 patients with abnormal and normal neurological outcomes in our dataset respectively. In order to balance the dataset, we therefore randomly select 11 patients from the 31 normal patients to make a balanced dataset for classification. This random selection of 11 patients among 31 patients with normal neurological outcomes is done ten times in each experiment and the mean and standard deviation of the classification accuracies are then calculated. Histograms of the four aforementioned features are normalized to the area below each histogram. These normalized histograms considered as feature vectors here are then fed to $k N N$ and random forest classifiers to classify SWIs to two normal and abnormal groups. In some experiments, we have used a combination of these feature vectors by concatenating all these feature vectors to constitute a long feature vector.

Table 1. Classification accuracy with selected features of vessels

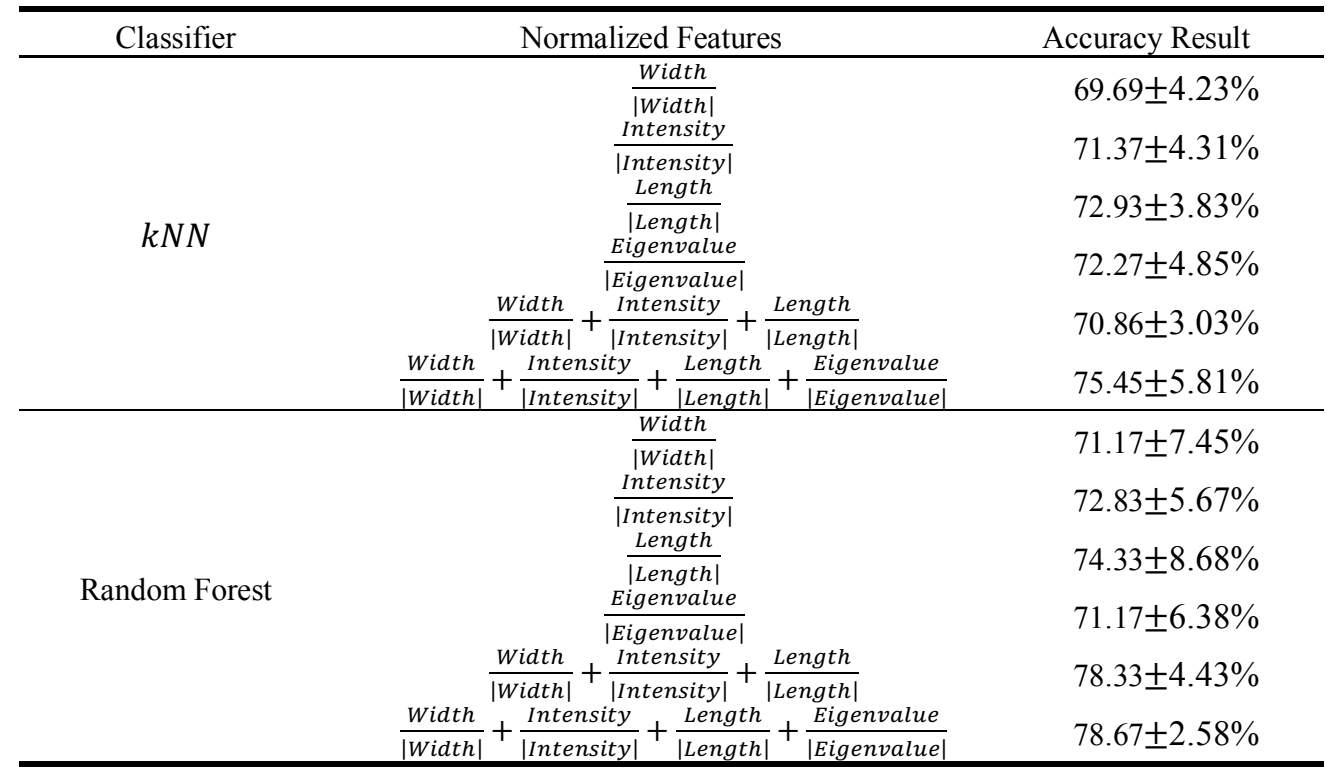


Table 2. The Pearson's Correlation Coefficient between the features

\begin{tabular}{ccccc}
\hline & Width & Intensity & Length & Eigenvalue \\
Width & 1 & 0.4831 & 0.1667 & -0.1714 \\
Intensity & 0.4831 & 1 & 0.4789 & -0.3122 \\
Length & 0.1667 & 0.4789 & 1 & -0.1973 \\
Eigenvalue & -0.1714 & -0.3122 & -0.1973 & 1 \\
\hline
\end{tabular}

As demonstrated in Table 1, the random forest classifier with four feature vectors (width, intensity, length and Hessian eigenvalue) produces the best accuracy $78.67 \pm 2.58 \%$. However, with a single feature vector, length feature enjoys higher accuracy than Hessian eigenvalue feature vector which has been used in [11]. Compared with only a single Hessian eigenvalue feature vector employed in [11], the new feature vectors we propose in this paper, produce higher classification accuracies for HIE outcome prognosis in SWIs. In Table 1, the strategy used for classification is leave-oneout cross validation for both classifiers. Figure 4(a to f) shows inter and intra class variations with various combinations of the four aforementioned features for two normal and abnormal groups in our dataset. In this figure, blue bars represent the histogram of distances between features from same groups (intra class variations), and brown bars are for the histogram of the distances between different groups (inter class variations). As shown in Figure 4(f), the overlap between two histograms is less than these histograms in Figure 4(a to d). This is in line with our results presented in Table 1 to indicate that the classification accuracies for a combination of features is higher than those for single features. Figure $4(\mathrm{~g})$ also shows the Cumulative Match Characteristic (CMC) Curve for the $k N N$ classifier. The highest accuracy among feature vectors is for the concatenated vectors of the four features, with $75.4 \%$ in rank 1 . It is noted that all the $k N N$ classification accuracies achieve $100 \%$ with rank 2 . Figure $4(\mathrm{~h})$ shows the ROC curve for various features and their combination in random forest classifier and for every feature or their combinations the area under the ROC curve is also presented in the figure. As observed from figure 4( $\mathrm{g}$ and $\mathrm{h}$ ), the performance of the system with the four aforementioned features, i.e. width, intensity, length and Hessian eigenvalue (blue curve) is the best. We have computed Pearson's correlation coefficient, $r$, that is calculated for all possible pairs of feature vectors as shown in Table 2. From table 2, we observe that some features are somewhat correlated. This explains why the classification results with $k N N$ for a combination width, intensity and length presents lower accuracy rate than the accuracy calculated by using only one of the features. To reduce the effect of dependency among features, we have therefore used random forest presenting better accuracy rate as shown in Table 1. 


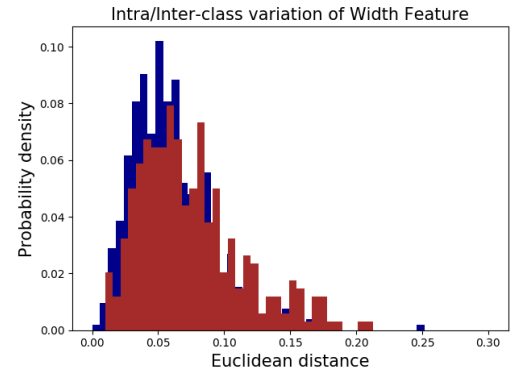

(a)

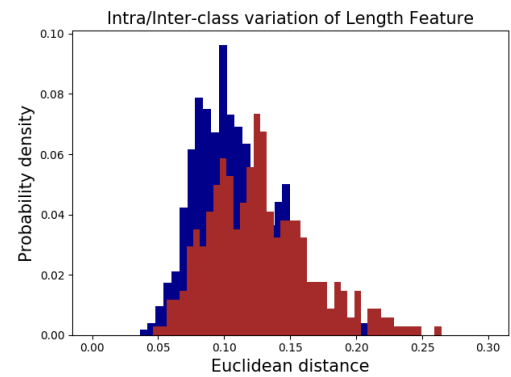

(c)

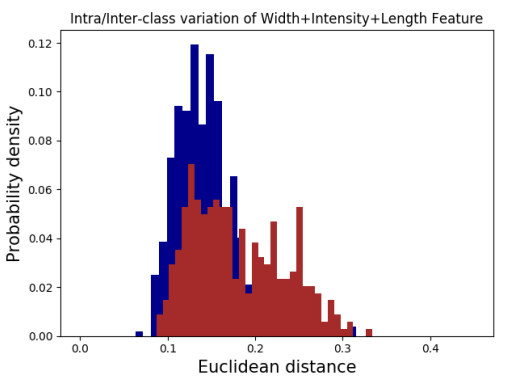

(e)

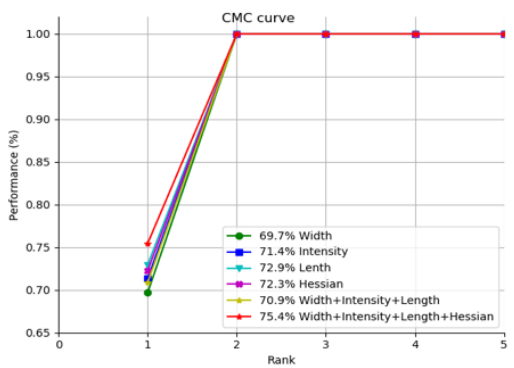

(g)

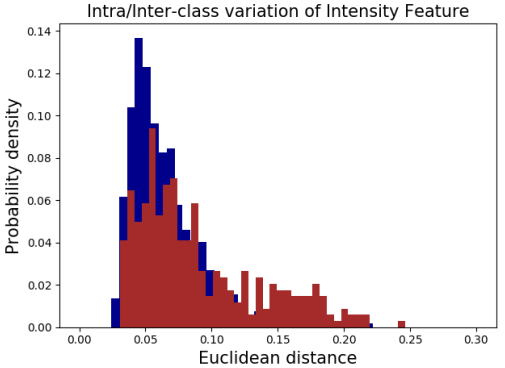

(b)

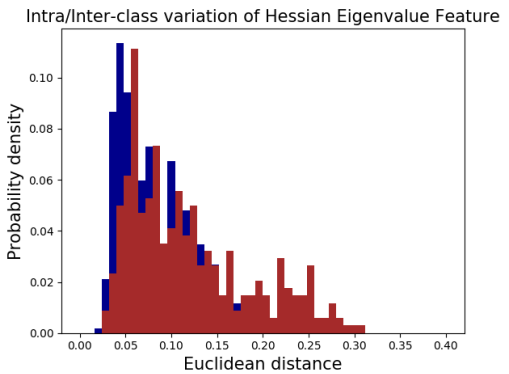

(d)

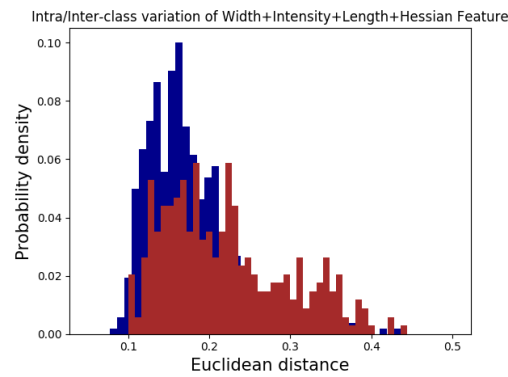

(f)

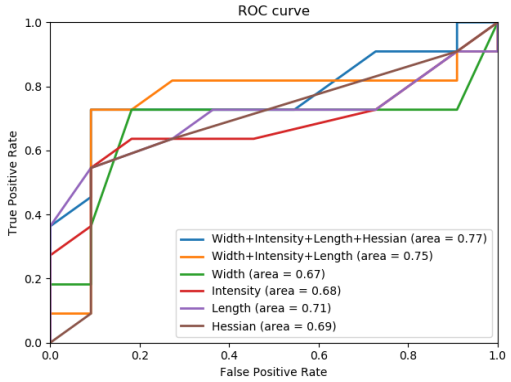

(h)

Fig. 4. (a f) Intra/inter-class variations for various features and their combinations $(\mathrm{g})$ Cumulative Match Characteristic (CMC) associated with $k N N$ classifier (h) Receiver Operating Characteristic (ROC) curve of our method with random forest classifier 


\section{$5 \quad$ Regressions Analysis}

HIE is associated with a high risk of cognitive and motor impairments in children [17]. Such impairments are evaluated by using the clinical outcomes of patients through a scoring system two years after the birth. It is therefore important to predict such clinical outcomes immediately after the birth. In our database, motor and cognitive development of infants with neonatal HIE are assessed by Bayley Scales at age 24 months, which result in a scaled score. The scores can either be used as a continuous variable or for which cut-offs of 'normal' or "delayed" can be applied. In normal population, both motor and cognitive score have a mean of 100 and standard deviation (SD) 15. A patient is considered to have a normal score, if the score is within 1standard deviation of mean. Mild delay is score equal or less than $1 \mathrm{SD}$, and moderate/severe delay is score more than 1.5 SD below the mean. We have 29 patients with cognitive scores and 28 with motor scores. All groups include patients with both normal and abnormal neurological outcome. There is no direct correlation between neurological outcomes and cognitive and motor scores. SVR is applied to find a regression model to predict the cognitive and motor scores respectively by using the four features vectors that we have extracted and a leave-one-out strategy is employed to evaluate the performance of the SVR regression method. By training the SVR model with the four feature vectors (as input, $x_{n}$ ) and cognitive or motor scores (as output, $y_{n}$ ), the mean absolute and mean relative score errors in our score calculations for all patients in our dataset can be predicted as shown in Table 3.

Table 3. SVR models with patients

\begin{tabular}{ccc}
\hline SVR Regressions & Mean Error & Mean Relative Error \\
29 Patients with cognitive scores & $11.40 \pm 13.24$ & $0.113 \pm 0.13$ \\
28 Patients with motor scores & $10.98 \pm 7.67$ & $0.109 \pm 0.067$ \\
\hline
\end{tabular}

In Table 3, each patient is given the predicted score by using the trained SVR. Errors are calculated for each test patient as absolute difference between the true scores of cognitive or motor and the corresponding predicted scores and we obtain a mean and a standard deviation for the errors. The error divided by the average value of scores across the data is also used to compute the relative value. As observed from table 3, the SVR regression method achieves better performance for predicting motor scores than predicting cognitive scores, i.e. relative value for patients with motor scores is 0.109 \pm 0.067 . However the mean relative errors for the cognitive scores is $0.113 \pm 0.13$ which is slightly higher than that for motor scores. It is expected that our regression errors decrease if we get more data with scores for clinical outcomes. 


\section{Conclusion and Future work}

In this paper, we examine the structure of and signal intensities of venous vessels to extract some features from SW images of infants for HIE outcome prognosis. We propose to use width, intensity and length to classify an infant into one of neurologically normal or abnormal groups. In our approach, we use the Snake Active Contour model to remove the background which is outside the brain tissue. Then we apply ridge detection method to obtain the ridge of vessels in order to enable us to compute histograms of width, intensity and length of vessels. To this end, we apply a vessel segmentation method to segment each vessel in each SW image with a separate label. We compute 1) the minimum Euclidean distance of the pixels on the vessel edge as width value, 2) the difference of the ridge point pixels intensity and edge pixels intensity as intensity value of the ridge and 3) the length of segmented ridges by counting the number of pixels labeled as a ridge. Meanwhile we also measure the eigenvalues of the Hessian matrix for all ridge pixels and their neighborhood and then normalize as well as concatenate all these features for classification. We have balanced our training dataset to avoid any classification bias. All four features and their combinations are fed to $k N N$ and random forest classifiers with a leave-one-out cross validation strategy. The concatenated features consisting of width, intensity, length and Hessian eigenvalue in random forest classifier present the best accuracy rate of $78.67 \pm 2.58 \%$. Our result is better than the classification accuracy reported in [11] which is $72.27 \pm 4.85 \%$. It is also noted that the data used in [11] is imbalanced and the classification in [11] has been achieved using the scale proposed by Kitamura [18]. However, in this paper, our HIE classification is carried out by using a balanced dataset and classification accuracies have been evaluated based on two neurologically normal and abnormal outcomes for infant patients. Finally, we train SVR model to predict cognitive and motor scores and use mean relative errors to measure the performance of the regression. Due to the lack of data, the lowest relative error of our SVR model is associated to motor scores, with mean relative error of $0.109 \pm 0.067$. We expect these results to improve if we get more data with the scores for clinical outcomes. As a result, our method improves the prognostic value of SWIs in HIE. The important future work is to determine what location of the brain has been damaged to be able to predict the behavioral abnormalities of the new born patients once they are grown up.

\section{References}

1. Friberg, H., \& Cronberg, T. (2017, February). Hypoxic-ischemic encephalopathy. In Seminars in neurology (Vol. 37, No. 01, pp. 003-004). Thieme Medical Publishers.

2. James, A., \& Patel, V. (2014). Hypoxic ischaemic encephalopathy. Paediatrics and Child Health, 24(9), 385-389.

3. Vannucci, R. C. (2000). Hypoxic-ischemic encephalopathy. American journal of perinatology, 17(03), 113-120.

4. Lally, P. J., Montaldo, P., Oliveira, V., Soe, A., Swamy, R., Bassett, P., ... \& Sashikumar, P. (2019). Magnetic resonance spectroscopy assessment of brain injury after moderate hypothermia in neonatal encephalopathy: a prospective multicentre cohort study. The Lancet Neurology, 18(1), 35-45. 
5. Bosemani, T., Poretti, A., \& Huisman, T. A. (2014). Susceptibility - weighted imaging in pediatric neuroimaging. Journal of Magnetic Resonance Imaging, 40(3), 530-544.

6. Liauw, L., Van der Grond, J., Van den Berg-Huysmans, A. A., Palm-Meinders, I. H., van Buchem, M. A., \& van Wezel-Meijler, G. (2008). Hypoxic-ischemic encephalopathy: diagnostic value of conventional MR imaging pulse sequences in term-born neonates. Radiology, 247(1), 204-212.

7. Obenaus, A., \& Ashwal, S. (2008). Magnetic resonance imaging in cerebral ischemia: focus on neonates. Neuropharmacology, 55(3), 271-280.

8. Ghosh, N., Sun, Y., Bhanu, B., Ashwal, S., \& Obenaus, A. (2014). Automated detection of brain abnormalities in neonatal hypoxia ischemic injury from MR images. Medical image analysis, 18(7), 1059-1069.

9. Murphy, K., van der Aa, N. E., Negro, S., Groenendaal, F., de Vries, L. S., Viergever, M. A., ... \& Išgum, I. (2017). Automatic quantification of ischemic injury on diffusion-weighted MRI of neonatal hypoxic ischemic encephalopathy. NeuroImage: Clinical, 14, 222-232.

10. Weiss, R. J., Bates, S. V., Song, Y. N., Zhang, Y., Herzberg, E. M., Chen, Y. C., ... \& Gollub, R. L. (2019). Mining multi-site clinical data to develop machine learning MRI biomarkers: application to neonatal hypoxic ischemic encephalopathy. Journal of translational medicine, 17(1), 1-16.

11. Wu, S., Mahmoodi, S., Darekar, A., Vollmer, B., Lewis, E., \& Liljeroth, M. (2017, July). Feature Extraction and Classification to Diagnose Hypoxic-Ischemic Encephalopathy Patients by Using Susceptibility-Weighted MRI Images. In Annual Conference on Medical Image Understanding and Analysis (pp. 527-536). Springer, Cham.

12. Barkovich, A. J., Hajnal, B. L., Vigneron, D., Sola, A., Partridge, J. C., Allen, F., \& Ferriero, D. M. (1998). Prediction of neuromotor outcome in perinatal asphyxia: evaluation of MR scoring systems. American Journal of Neuroradiology, 19(1), 143-149.

13. Citraro, L., Mahmoodi, S., Darekar, A., \& Vollmer, B. (2017). Extended three-dimensional rotation invariant local binary patterns. Image and vision Computing, 62, 8-18.

14. Kass, M., Witkin, A., \& Terzopoulos, D. (1988). Snakes: Active contour models. International journal of computer vision, 1(4), 321-331.

15. Wu, K., Otoo, E., \& Shoshani, A. (2005, April). Optimizing connected component labeling algorithms. In Medical Imaging 2005: Image Processing (Vol. 5747, pp. 1965-1976). International Society for Optics and Photonics.

16. Fiorio, C., \& Gustedt, J. (1996). Two linear time union-find strategies for image processing. Theoretical Computer Science, 154(2), 165-181.

17. van Schie, P. E., Schijns, J., Becher, J. G., Barkhof, F., van Weissenbruch, M. M., \& Vermeulen, R. J. (2015). Long-term motor and behavioral outcome after perinatal hypoxic-ischemic encephalopathy. European Journal of Paediatric Neurology, 19(3), 354-359.

18. Kitamura, G., Kido, D., Wycliffe, N., Jacobson, J. P., Oyoyo, U., \& Ashwal, S. (2011). Hypoxic-ischemic injury: utility of susceptibility-weighted imaging. Pediatric neurology, 45(4), 220-224. 